\title{
Chaotic Behavior Analysis of a New Incommensurate Fractional-Order Hopfield Neural Network System
}

\author{
Nadjette Debbouche, ${ }^{1}$ Adel Ouannas, ${ }^{2}$ Iqbal M. Batiha ${ }^{(D)},{ }^{3,4}$ Giuseppe Grassi, ${ }^{5}$ \\ Mohammed K. A. Kaabar $\mathbb{D}^{6,7,7,8}$ Hadi Jahanshahi ${ }^{\mathbb{D}},{ }^{9}$ Ayman A. Aly, ${ }^{10}$ \\ and Awad M. Aljuaid ${ }^{11}$ \\ ${ }^{1}$ Department of Mathematics and Computer Science, University of Larbi Ben M'hidi, Oum El Bouaghi, Algeria \\ ${ }^{2}$ Laboratory of Dynamical Systems and Control, University of Larbi Ben M'hidi, 04000 Oum El Bouaghi, Algeria \\ ${ }^{3}$ Department of Mathematics, Faculty of Science and Technology, Irbid National University, 2600 Irbid, Jordan \\ ${ }^{4}$ Nonlinear Dynamics Research Center (NDRC), Ajman University, 346 Ajman, UAE \\ ${ }^{5}$ Dipartimento Ingegneria Innovazione, Universita del Salento, 73100 Lecce, Italy \\ ${ }^{6}$ Gofa Camp, Near Gofa Industrial College and German Adebabay, Nifas Silk-Lafto, 26649 Addis Ababa, Ethiopia \\ ${ }^{7}$ Jabalia Camp, United Nations Relief and Works Agency (UNRWA), Palestinian Refugee Camp, Gaza Strip, \\ Jabalya, State of Palestine \\ ${ }^{8}$ Institute of Mathematical Sciences, Faculty of Science, University of Malaya, Kuala Lumpur 50603, Malaysia \\ ${ }^{9}$ Department of Mechanical Engineering, University of Manitoba, Winnipeg R3T 5V6, Canada \\ ${ }^{10}$ Department of Mechanical Engineering, College of Engineering, Taif University, P.O. Box 11099, Taif 21944, Saudi Arabia \\ ${ }^{11}$ Department of Industrial Engineering, College of Engineering, Taif University, P.O. Box 11099, Taif 21944, Saudi Arabia
}

Correspondence should be addressed to Mohammed K. A. Kaabar; mohammed.kaabar@wsu.edu

Received 3 May 2021; Revised 6 September 2021; Accepted 7 October 2021; Published 8 November 2021

Academic Editor: Miaomiao Wang

Copyright ( $\odot 2021$ Nadjette Debbouche et al. This is an open access article distributed under the Creative Commons Attribution License, which permits unrestricted use, distribution, and reproduction in any medium, provided the original work is properly cited.

\begin{abstract}
This study intends to examine different dynamics of the chaotic incommensurate fractional-order Hopfield neural network model. The stability of the proposed incommensurate-order model is analyzed numerically by continuously varying the values of the fractional-order derivative and the values of the system parameters. It turned out that the formulated system using the Caputo differential operator exhibits many rich complex dynamics, including symmetry, bistability, and coexisting chaotic attractors. On the other hand, it has been detected that by adapting the corresponding controlled constants, such systems possess the so-called offset boosting of three variables. Besides, the resultant periodic and chaotic attractors can be scattered in several forms, including 1D line, 2D lattice, and 3D grid, and even in an arbitrary location of the phase space. Several numerical simulations are implemented, and the obtained findings are illustrated through constructing bifurcation diagrams, computing Lyapunov exponents, calculating Lyapunov dimensions, and sketching the phase portraits in 2D and 3D projections.
\end{abstract}

\section{Introduction}

The artificial neural networks, which are deemed one of the deepest learning technologies that are included under the rubric of the so-called artificial intelligence, have recently received a considerable amount of interest by many researchers whose practical work associates with the human brain [1]. In order to make an effective progress with the development of modern engineering and electronics, it is necessary to continuously attempt to improve this intelligent scheme. In 1943, McCulloch and Pitts are the first scientists who studied the artificial neural networks [2]. In conformity with their investigation, numerous benign engineering and electronic applications have been employed in several applied fields. For instance, switching in electronic circuits, the oscillation of systems in accordance with the impact of an 
earthquake, image and signal processing, impacting machines, power circuits, and dry friction are some of such applications [3]. In 1982, with the aim of dealing with some optimization and computational issues and to conquer some specific problems associated with the hardware's execution, a novel memory neural network was established by Hopfield called later on the Hopfield neural network (HNN) [4]. Presently, this type of networks has begun taking its place in different industrial sectors, motivating a lot of investigators to further explore the dynamical properties for its states and moreover deduce other ones [5].

Due to the key role of using the fractional calculus in formulating many phenomena rather than that of using the classical calculus, the HNNs were fractionalized to be later on called the fractional-order Hopfield neural networks (FoHNNs) [6, 7]. The basic idea of the FoHNNs' origin may be owed to Boroomand and Menhaj who carried out a replacement of the fractance instead of the ordinary capacitor within the classical HNN model [8]. The key benefit of such replacement refers to the truth that the fractionalorder derivatives can describe the HNN more efficiently due to the infinite memory and some other hereditary properties that can be generated from its various processes [9]. From a different point of view, the inclusion process of a memory term into the HNN model by incorporating the fractionalorder derivatives/integrals can provide a super calculation capability, which might be needed in, e.g., the stimulus anticipation and the information processing as well as other calculations associated with the oscillatory neuronal firing [10]. For these reasons and more, the analysis of FoHNNs is recently considered one of the main promising topics that benefits future researchers in different applied science fields.

Several significant numerical findings related to the presence of the chaotic behavior and the limit cycles for the dynamics of the FoNN are discussed in the literature. For instance, the stability of the FoHNN was fully investigated through energy-like function analysis in [8]. However, a theoretical approach, based on the harmonic balance theory, was used to investigate the existence of chaos for a cellular neural network model in [11]. A chaos neuron model was proposed and examined as a novel artificial neuron model in [12]. Moreover, in [13], a fractional-order cellular neural network model was introduced by replacing the traditional first-order cell by the fractional-order one. The chaotic synchronization of such networks was also discussed in several other papers. We find certain chaotic behaviors in the time-delayed FoNN system, studied well in [14]. Also, the chaos feedback control and synchronization systems were constructed for a neuron network system by Zhou et al. in [15]. One year later, Zhou et al. once again illustrated the chaotic synchronization system for a FoNN system in [16]. In [17], the Laplace transform and the generalized Gronwall inequality were employed to examine the FoHNN models in terms of its finite-time stability, whereas various dynamic features like constructing bifurcation diagrams, chaos, stability, and multistability of the FoNNs were studied in [9]. In [18], an $\alpha$-synchronization and an $\alpha$-stability were determined and explored in the FoNN models, whereas a uniform stability of such models was described and analyzed with time delay in [19] with the help of using an efficient kind of error norm. Similarly, the complex dynamics of several modern maps have been recently studied such as the initialdependent extreme multistability and offset-boosted coexisting attractor.

In light of the several applications of the FoHNN models in different applied science fields and in order to move forward in further discovery of more properties of the FoHNNs dynamics, this paper attempts to study different chaotic dynamics of such networks with incommensurate order. Besides, it intends to analyze the stability of the proposed system numerically by continuous varying the fractional-order derivative values as well as the values of system parameters. Such analysis will be carried out by performing several numerical simulations, like constructing the bifurcation diagrams, computing Lyapunov exponents, calculating Lyapunov dimensions, and sketching the phase portraits in 2D and 3D projections. Several rich complex dynamics, including symmetry, bistability, and coexisting chaotic attractors will be investigated and discussed. It will be also shown, through adapting some controlled constants, that the proposed system will possess the offset boosting of three variables. However, the structure of this article is arranged in the following manner: Section 2 will study and discuss different complex dynamics of the incommensurate FoHNN model through illustrating their different corresponding numerical simulations. Dynamics of the incommensurate fractional-order model will be discussed in Section 3. The variable-boostable attractors that are generated from the incommensurate-order model will be discussed and analyzed in Section 4, followed by Section 5 that will summarize the whole work.

\section{Preliminaries and Mathematical Model}

This part intends to describe some essential fundamentals in regard to the fractional calculus, especially the Caputo differential operator and the Riemann-Liouville integral operator which were reported in [20]. Besides, a new version of the HNN model will be proposed in light of its incommensurate fractional-order derivatives.

Definition 1. The Riemann-Liouville fractional-order integral operator of the function $h$ can be expressed as

$$
I^{\delta} h(t)=\frac{1}{\Gamma(\delta)} \int_{0}^{t} \frac{h(\tau)}{(t-\tau)^{(1-\delta)}} \mathrm{d} \tau,
$$

where $h \in C^{m}(0, T], \delta>0, T>0$, and $m \in \mathbb{N}$.

Definition 2. The Caputo fractional-order differential operator of the function $h$ can be expressed as

$$
D^{\delta} h(t)= \begin{cases}\frac{1}{\Gamma(m-\delta)} \int_{0}^{t}(t-\tau)^{m-\delta-1} h^{(m)}(\tau) \mathrm{d} \tau, & \delta \in(m-1, m), \\ h^{(m)}(t), & \delta=m,\end{cases}
$$

where $h \in C^{m}(0, T], T>0, m \in \mathbb{N}$, and $\delta \in[m-1, m]$. 
In [9], a mathematical model of FoHNN with a ring structure was established and its stability analysis was discussed in view of some features of its parameters. The model was formulated by the following three-dimensional system:

$$
\left\{\begin{array}{l}
D^{q} x(t)=-x(t)+2 \sin (x(t))+a \sin (y(t))+b \sin (z(t)), \\
D^{q} y(t)=-y(t)+b \sin (x(t))+2 \sin (y(t))+a \sin (z(t)), \\
D^{q} z(t)=-z(t)+a \sin (x(t))+b \sin (y(t))+2 \sin (z(t)),
\end{array}\right.
$$

where $a$ and $b$ are the system parameters, $D^{q}$ is the Caputo differential operator of order $q$, and $x, y$, and $z$ are the states of the system. As a matter of fact, Kaslik and Sivasundaram studied and analyzed the stability of the above system by taking its fractional-order derivatives in commensurateorder case [9]. To illustrate the chaotic motion of this neural network system in its integer-order case (i.e., system (4) when $q=1$ ), we plot Figure 1 that shows its phase portraits in different planes generated by its states. The basin of attraction of this integer-order system is also shown in Figure 2, where the initial conditions (ICs) can be shown in yellow and blue regions, leading to the limit cycles and to the chaotic attractors, respectively.

In this work, we assert that if we change these derivatives to be in its incommensurate-order case, then such systems will exhibit more rich complex dynamics and more chaotic patterns than in the previous model reported in [9]. Thus, the new incommensurate fractional-order version of the previous HNN model, which will be considered from now on, can be formulated as follows:

$$
\left\{\begin{array}{l}
D^{q_{1}} x(t)=-x(t)+2 \sin (x(t))+a \sin (y(t))+b \sin (z(t)), \\
D^{q_{2}} y(t)=-y(t)+b \sin (x(t))+2 \sin (y(t))+a \sin (z(t)), \\
D^{q_{3}} z(t)=-z(t)+a \sin (x(t))+b \sin (y(t))+2 \sin (z(t)),
\end{array}\right.
$$

where $a, b, x, y$, and $z$ are defined above, while $D^{q_{i}}$ is the Caputo differential operator of order $q_{i}$ such that $0<q_{i} \leq 1$ and $i=1,2,3$. Actually, in order to solve the above system, one can implement the predictor-corrector method which was proposed by Diethelm et al. in [21]. Although this method can provide an accurate solution of a given nonlinear fractional-order system numerically [22], an enhanced approach called Adams-Bashforth-Moulton scheme was established in $[23,24]$.

\section{Dynamics of the Incommensurate Fractional- Order Model}

In this section, different complex dynamics of incommensurate FoHNN model (4) will be numerically studied and analyzed, including discussion of the stability analysis versus taking different values of incommensurate fractional-order derivatives and also of system parameters and presenting some special phenomena that could be generated from the proposed model such as presenting the so-called symmetry and coexisting attractors. For this purpose, several numerical tools will be used for performing some required simulations, including constructing bifurcation diagrams, sketching the phase portraits of the system dynamics in $2 \mathrm{D}$ and $3 \mathrm{D}$ projections, plotting the basin of attractions, and also computing Lyapunov exponents/dimensions.

3.1. Stability vs. Different Incommensurate Fractional-Order Derivatives. Here, we will take the two parameters of the system as $a=1$ and $b=-9$ as well as we will consider the IC of the states of system $(4)$ as $\left(x_{0}, y_{0}, z_{0}\right)=(-1,1,1)$. In this part, we aim to study the stability of model (4) according to the following three cases: the first one is carried out by fixing $q_{2}=$ $q_{3}=1$ and continuously varying the value of $q_{1}$, the second one is performed by fixing $q_{1}=q_{2}=1$ and continuously varying the value of $q_{2}$, and finally, the third one is implemented by fixing $q_{1}=q_{2}=1$ and continuously varying the value of $q_{3}$. Accordingly, the bifurcation diagrams and Lyapunov exponents for such three cases are plotted in Figures 3-5, respectively. Based on these figures, one can observe the existence of positive Lyapunov exponents, confirming that FoHNN system (4) shows chaotic behavior. In addition, one might notice, from such figures again, that system (4) is asymptotically stable to a limit cycle when $q_{1}, q_{2}, q_{3} \in(0.6,0.73)$, while it begins losing its stability and begins behaving in a chaotic mode when $q_{1} \in(0.82,1), q_{2} \in(0.83,1)$, and $q_{3} \in(0.84,1)$. It might be further noticed that once the values of the incommensurate fractional-order derivatives are increased, different periodic windows will be shown. For instance, to deal with the aforesaid three cases, we may take the values of the fractional-order derivatives as $q_{1}=0.89$ and $q_{2}=q_{3}=1$, which, directly, implies the three Lyapunov exponents, $\mathrm{LE}_{1}=0.26, \mathrm{LE}_{2}=0$, and $\mathrm{LE}_{3}=-4.39$, with $|0.26|<|-4.398|$. On the other hand, if one takes $q_{2}=0.90$ and $q_{1}=q_{3}=1$, then the following three Lyapunov exponents will be yielded: $\mathrm{LE}_{1}=0.51, \mathrm{LE}_{2}=0$, and $\mathrm{LE}_{3}=-2.63$, with $|0.51|<|-2.63|$. Finally, taking $q_{3}=0.87$ and $q_{1}=q_{2}=1$ gives the following three Lyapunov exponents: $\mathrm{LE}_{1}=0.31, \mathrm{LE}_{2}=0$, and $\mathrm{LE}_{3}=-5.06$, with $|0.31|<|-5.06|$. Consequently, the corresponding Lyapunov dimension, which is considered an estimation of the complexity of the generated attractor from the system, can be calculated according to the previous three cases by considering the following formula:

$$
D_{K Y}=j+\frac{1}{\left|\mathrm{LE}_{j+1}\right|} \sum_{i=1}^{j} \mathrm{LE}_{i}
$$

where $j$ is the greatest integer number that satisfies $\sum_{i=1}^{j} \mathrm{LE}_{i} \geq 0$ and $\sum_{i=1}^{j+1} \mathrm{LE}_{i}<0$. In other words, the calculated Lyapunov dimensions of system (4) according to the previous values of incommensurate fractional-order derivatives are $D_{K Y}=2.09>2, D_{K Y}=2.19>2$, and $D_{K Y}=2.06>2$, respectively. This, consequently, leads us to deduce that there is a chaotic attractor of system (4). To see this, Figures 6-8 present the complex chaotic attractor of system (4) on different $2 \mathrm{D}$ projections according to the three considered cases: $\quad\left(q_{1}=0.89\right.$ and $\left.q_{2}=q_{3}=1\right), \quad\left(q_{2}=0.90 \quad\right.$ and $\left.q_{1}=q_{3}=1\right)$, and $\left(q_{3}=0.87\right.$ and $\left.q_{1}=q_{2}=1\right)$, respectively. In addition, Figures 9-11 exhibit the corresponding 3D projections of the chaotic attractors that are generated by system (4). 


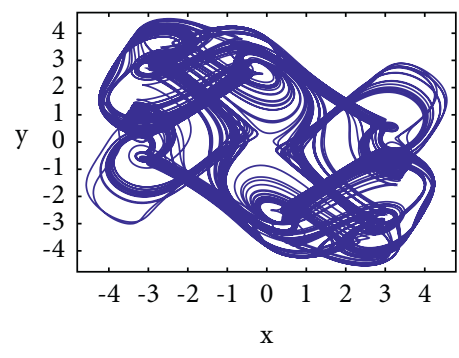

(a)

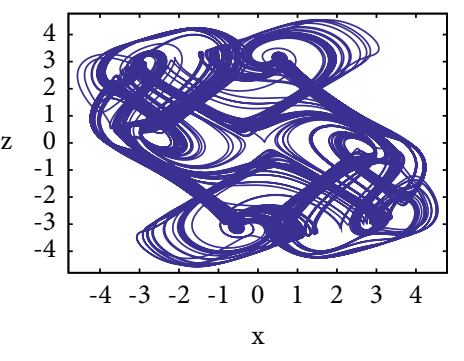

(b)

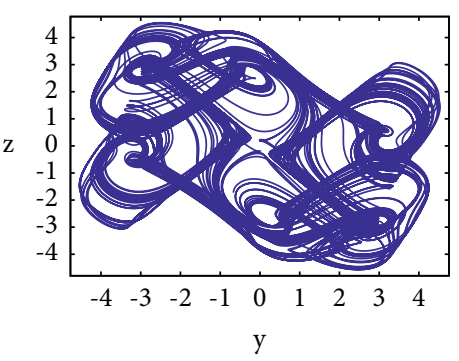

(c)

Figure 1: Chaotic attractor of system (3) when $q=1$ according to the system parameters $a=1$ and $b=-9$ and the IC $\left(x_{0}, y_{0}, z_{0}\right)=$ $(-1,1,1)$ on (a) $x y$ plane, (b) $x z$ plane, and (c) $y z$ plane.

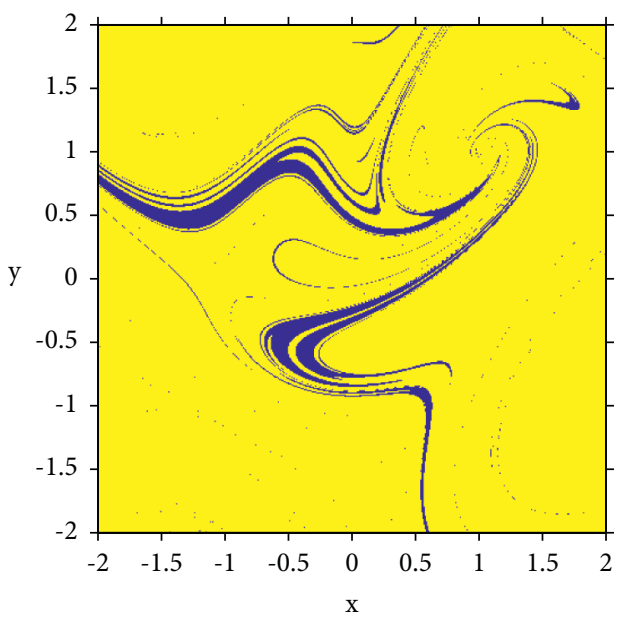

Figure 2: Basin of attraction of system (3) when $q=1$ according to the system parameters $a=1$ and $b=-9$ and to the initial condition of the third state variable $z=0$.

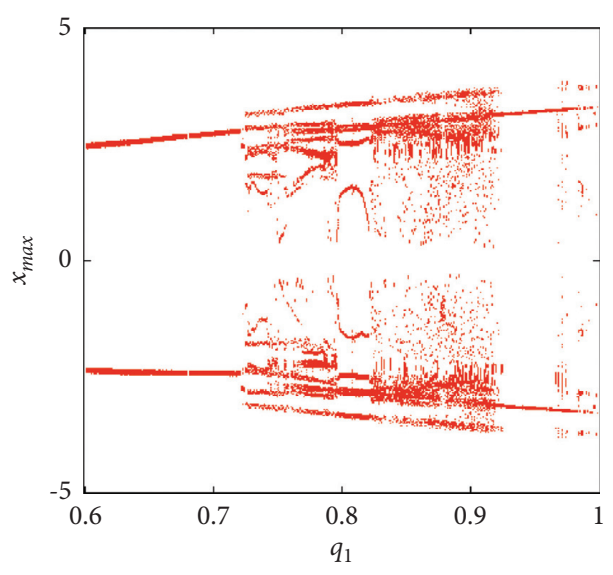

(a)

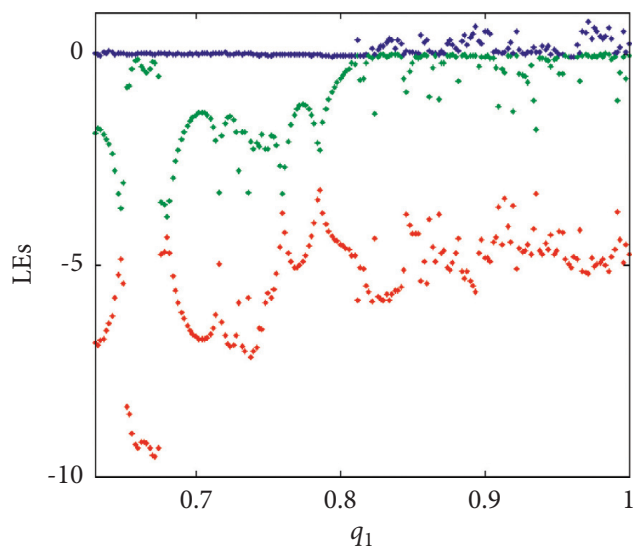

(b)

Figure 3: (a) The diagram of bifurcation. (b) Lyapunov exponents of system (4) when $q_{1} \in(0.6,1)$ and $q_{2}=q_{3}=1$ according to the system parameters $a=1$ and $b=-9$ and the IC $\left(x_{0}, y_{0}, z_{0}\right)=(-1,1,1)$.

3.2. Stability vs. Different Values of the System Parameters. This section will explore the stability of system (4) by continuously varying the values of the system parameters $a$ and $b$ and by fixing the incommensurate fractional-order derivative values at $\left[q_{1}, q_{2}, q_{3}\right]=[0.8,0.90,0.91]$ as well as fixing the IC at $\left(x_{0}, y_{0}, z_{0}\right)=(-1,1,1)$. Immediately, Figures 12 and 13 represent the bifurcation diagrams together with the Lyapunov exponents of system (4) when $a \in(0,5)$ and $b \in(-20,0)$, respectively. One might observe based on such plots that when the values of the system parameters are 


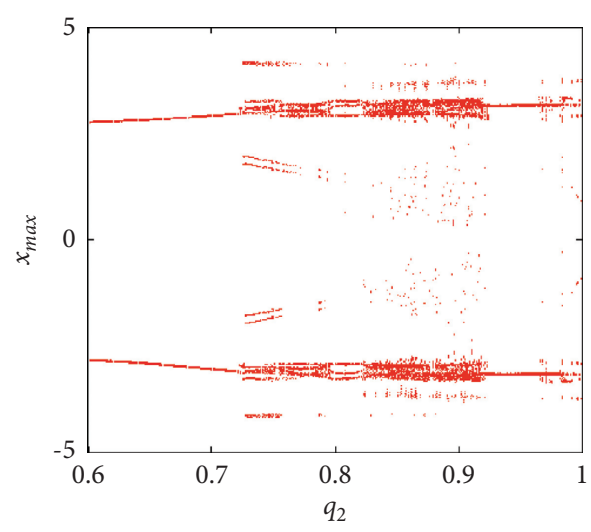

(a)

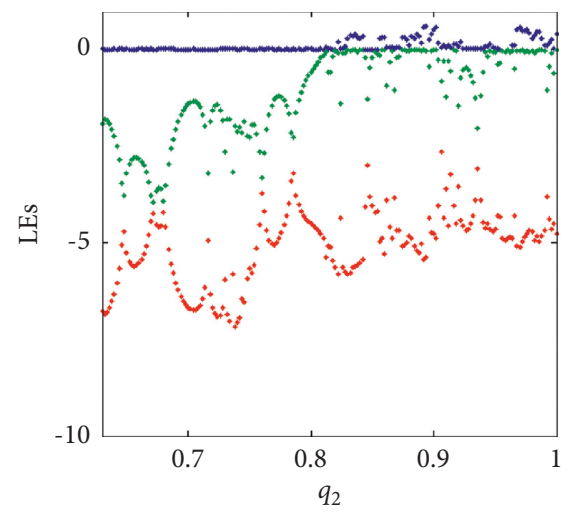

(b)

FIgURE 4: (a) The diagram of bifurcation. (b) Lyapunov exponents of system (4) when $q_{2} \in(0.6,1)$ and $q_{1}=q_{3}=1$ according to the system parameters $a=1$ and $b=-9$ and the IC $\left(x_{0}, y_{0}, z_{0}\right)=(-1,1,1)$.

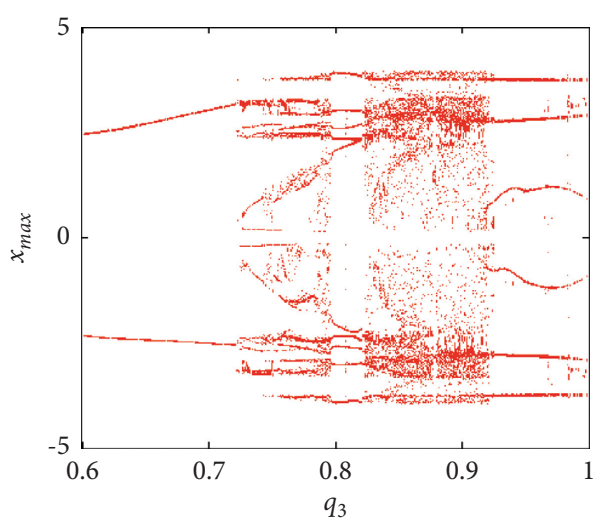

(a)

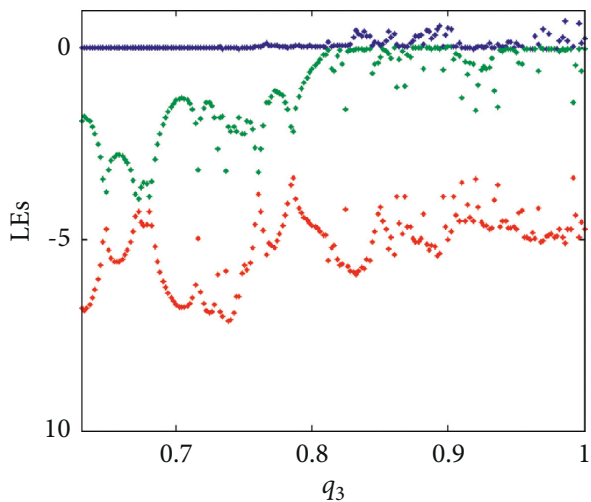

(b)

Figure 5: (a) The diagram of bifurcation. (b) Lyapunov exponents of system (4) when $q_{1} \in(0.6,1)$ and $q_{2}=q_{3}=1$ according to the system parameters $a=1$ and $b=-9$ and the IC $\left(x_{0}, y_{0}, z_{0}\right)=(-1,1,1)$.

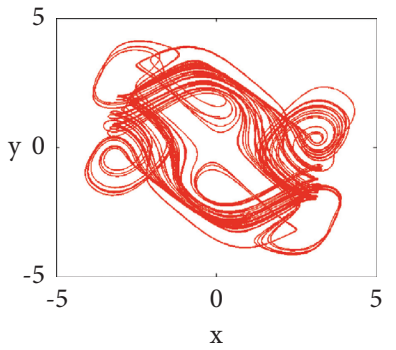

(a)

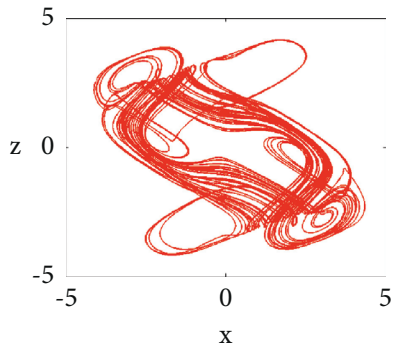

(b)

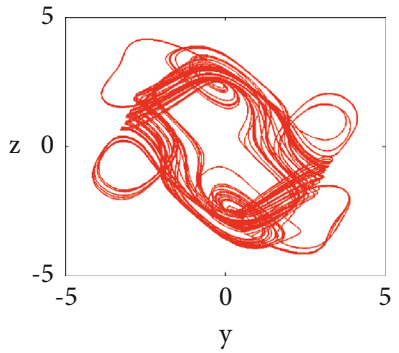

(c)

FIgURE 6: Chaotic attractor of system (4) when $q_{1}=0.89$ and $q_{2}=q_{3}=1$ according to the system parameters $a=1$ and $b=-9$ and the IC $\left(x_{0}, y_{0}, z_{0}\right)=(-1,1,1)$ on (a) $x y$ plane, (b) $x z$ plane, and (c) $y z$ plane.

decreased, system (4) will be asymptotically stable and then it will exhibit periodic oscillations to chaos. Moreover, the chaotic ranges with periodic windows will be of $a \in(0,2.9)$ and $b \in(-20,-7.5)$. Obviously, there exist certain positive Lyapunov exponents within these ranges, confirming the chaotic behavior of system (4).
3.3. Symmetry, Bistability, and Coexisting Chaotic Attractors. Symmetric dynamical systems are typically obtained when they often exhibit a symmetric pair of coexisting attractors. This property has attracted considerable interest in the field of nonlinear dynamic systems. To obtain a complete overview of this property, the reader may refer to [25]. In regard 


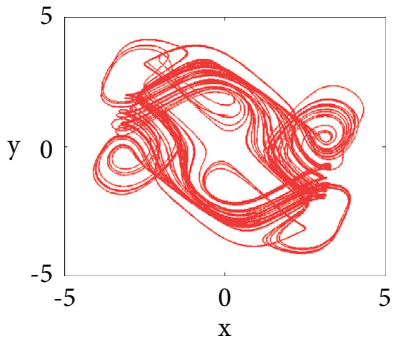

(a)

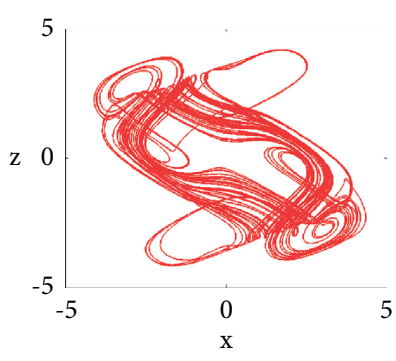

(b)

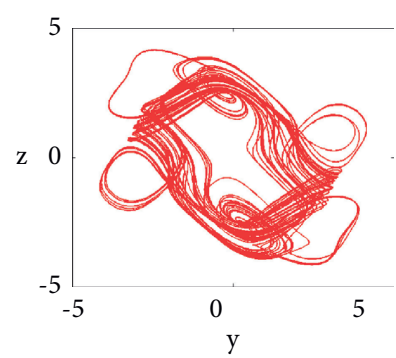

(c)

FIgURe 7: Chaotic attractor of system (4) when $q_{2}=0.90$ and $q_{1}=q_{3}=1$ according to the system parameters $a=1$ and $b=-9$ and the IC $\left(x_{0}, y_{0}, z_{0}\right)=(-1,1,1)$ on (a) $x y$ plane, (b) $x z$ plane, and (c) $y z$ plane.

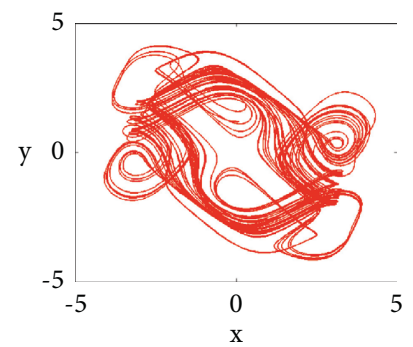

(a)

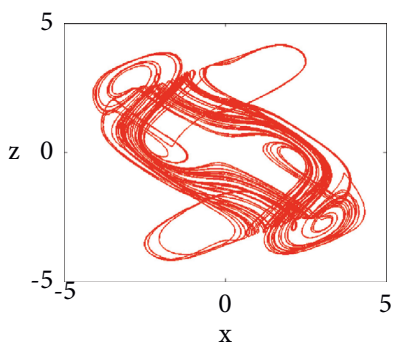

(b)

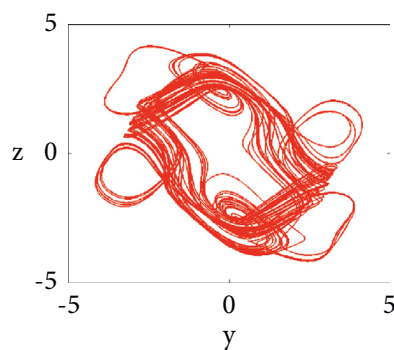

(c)

FIGURE 8: Chaotic attractor of system (4) when $q_{3}=0.87$ and $q_{1}=q_{2}=1$ according to the system parameters $a=1$ and $b=-9$ and the IC $\left(x_{0}, y_{0}, z_{0}\right)=(-1,1,1)$ on (a) $x y$ plane, (b) $x z$ plane, and (c) $y z$ plane.

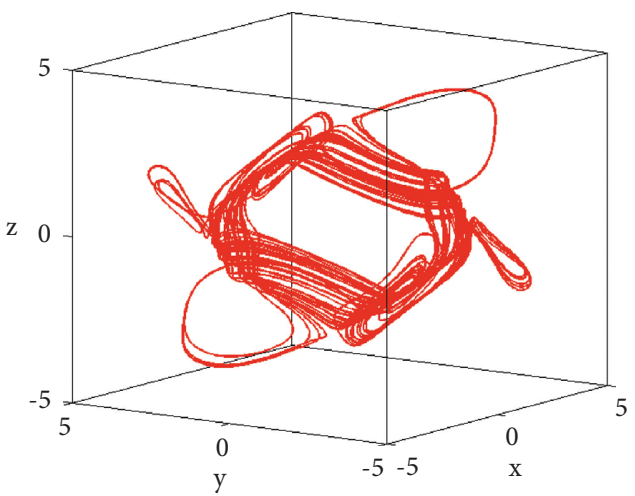

FIGURE 9: Chaotic attractor of system (4) on 3D projections when $q_{1}=0.89$ and $q_{2}=q_{3}=1$ according to the system parameters $a=1$ and $b=-9$ and the IC $\left(x_{0}, y_{0}, z_{0}\right)=(-1,1,1)$.

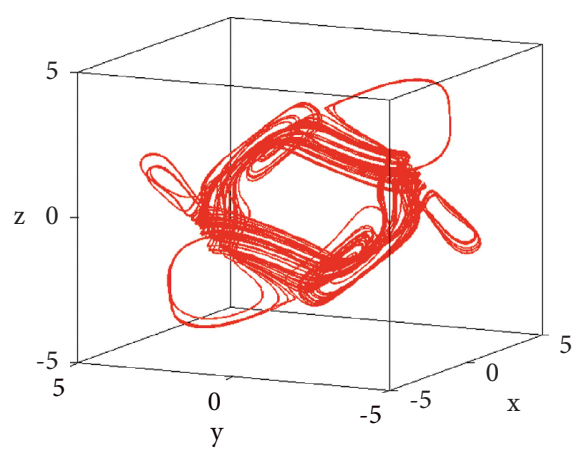

Figure 10: Chaotic attractor of system (4) on 3D projections when $q_{2}=0.90$ and $q_{1}=q_{3}=1$ according to the system parameters $a=1$ and $b=-9$ and the IC $\left(x_{0}, y_{0}, z_{0}\right)=(-1,1,1)$.

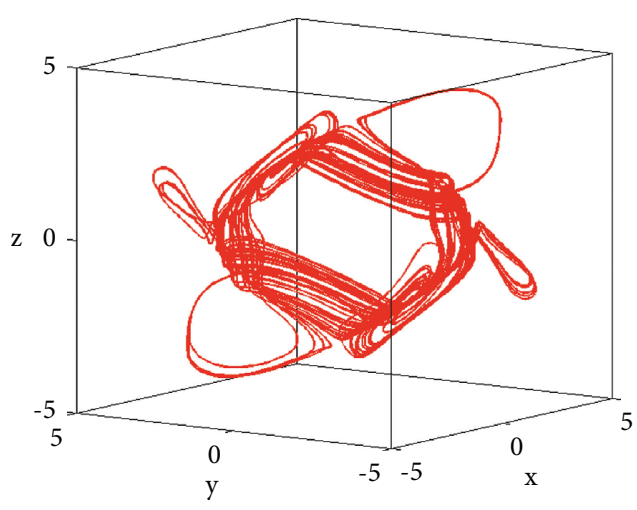

FIgURE 11: Chaotic attractor of system (4) on 3D projections when $q_{3}=0.87$ and $q_{1}=q_{2}=1$ according to the system parameters $a=1$ and $b=-9$ and the IC $\left(x_{0}, y_{0}, z_{0}\right)=(-1,1,1)$.

to our study, we will assume that the system parameters are $a=1$ and $b=-9$ and we will select two ICs as $\left(x_{0}, y_{0}, z_{0}\right)=$ $(1,1,1)$ for the red trajectory and $\left(x_{0}, y_{0}, z_{0}\right)=(-1,-1,-1)$ for the blue trajectory. In light of these values, we observe that system (4) will, e.g., generate a symmetric pair of coexisting limit cycles when $\left[q_{1}, q_{2}, q_{3}\right]=[0.70,1,1]$ (see Figure 14(a)), while it will generate a symmetric pair of coexisting periodic attractors when $\left[q_{1}, q_{2}, q_{3}\right]=[0.80,1,1]$ (see Figure 14(b)), and moreover, it will generate a symmetric pair of coexisting chaotic attractors when $\left[q_{1}, q_{2}, q_{3}\right]=[0.89,1,1]$ (see Figure $14(\mathrm{c})$ ).

On the other hand, the bistability property, which is deemed one of the most recent dynamic phenomena of a system, has attracted many researchers in recent years. 


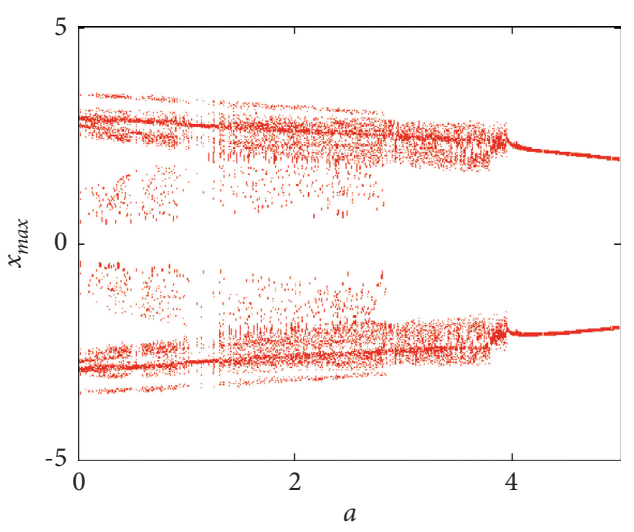

(a)

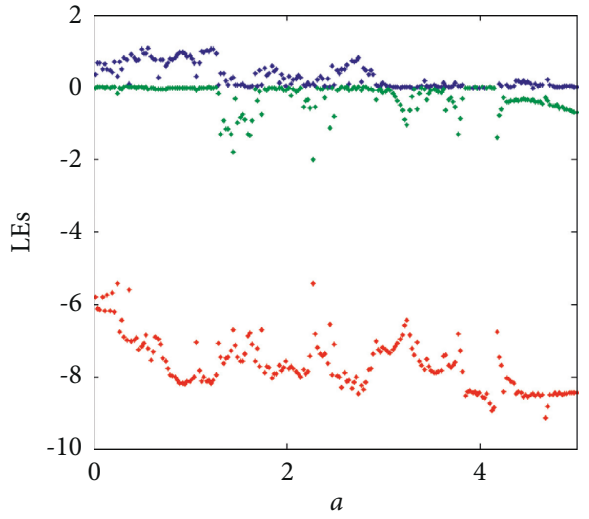

(b)

Figure 12: (a) The diagram of bifurcation. (b) Lyapunov exponents of system (4) when $a \in(0,5)$ according to the other system parameter $b=-9$, the incommensurate fractional order $\left[q_{1}, q_{2}, q_{3}\right]=[0.8,0.90,0.91]$, and the IC $\left(x_{0}, y_{0}, z_{0}\right)=(-1,1,1)$.

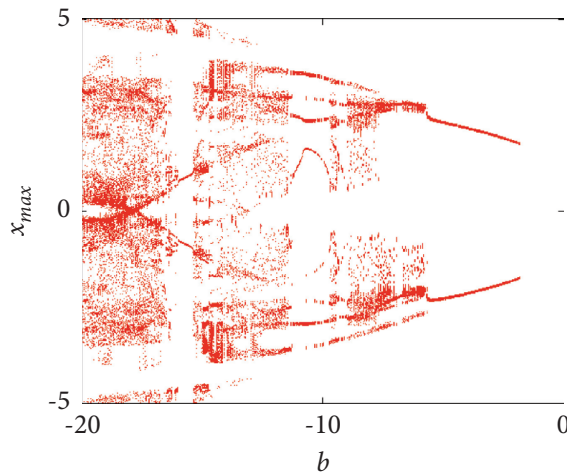

(a)

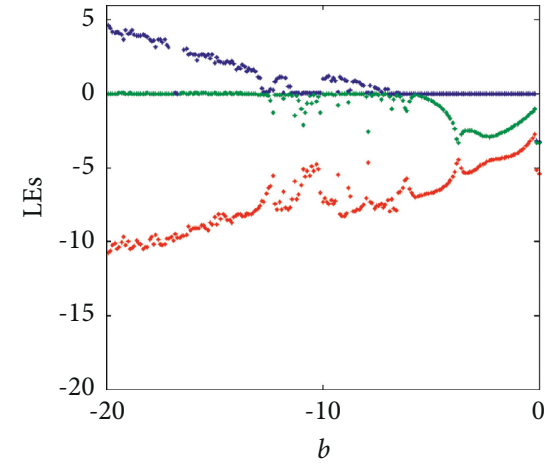

(b)

FiguRE 13: (a) The diagram of bifurcation. (b) Lyapunov exponents of system (4) when $b \in(-20,0)$ according to the other system parameter $a=1$, the incommensurate fractional order $\left[q_{1}, q_{2}, q_{3}\right]=[0.8,0.90,0.91]$, and the IC $\left(x_{0}, y_{0}, z_{0}\right)=(-1,1,1)$.

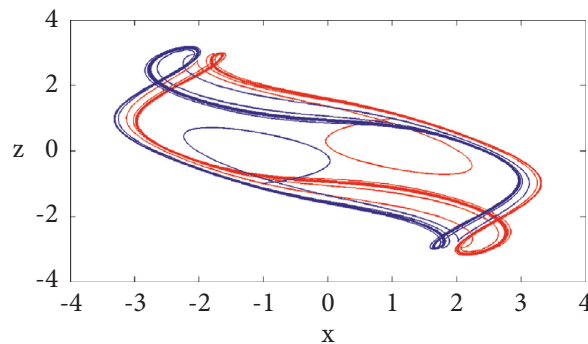

(a)

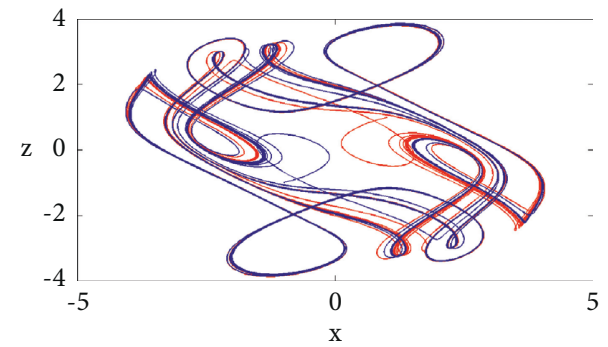

(b)

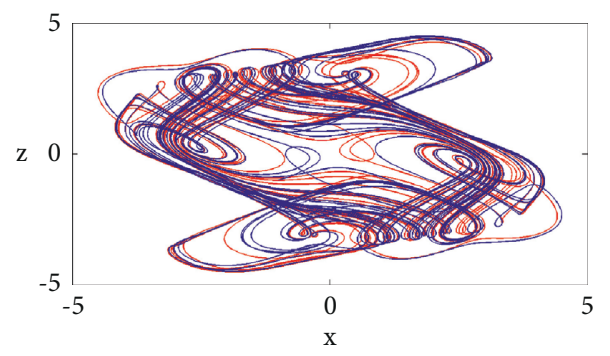

(c)

FIGURE 14: Phase portraits of coexisting symmetric attractors according to the system parameters $a=1$ and $b=-9$, the IC $\left(x_{0}, y_{0}, z_{0}\right)=(1,1,1)$ for the red line, and the IC $\left(x_{0}, y_{0}, z_{0}\right)=(-1,-1,-1)$ for the blue line by continuously varying $q_{i}$ as $(\mathrm{a})\left[q_{1}, q_{2}, q_{3}\right]=[0.70,1,1]$, (b) $\left[q_{1}, q_{2}, q_{3}\right]=[0.80,1,1]$, and (c) $\left[q_{1}, q_{2}, q_{3}\right]=[0.89,1,1]$. 


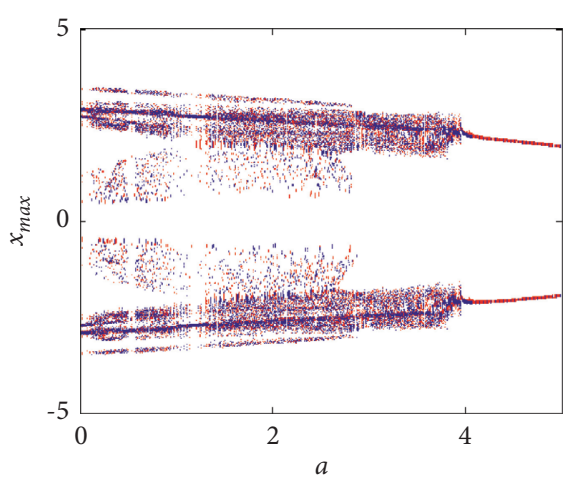

(a)

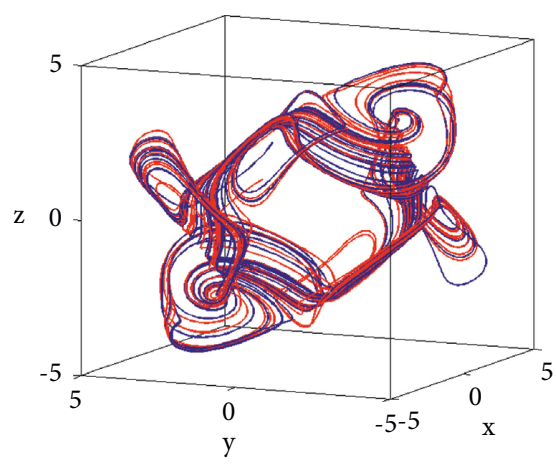

(b)

Figure 15: (a) Bifurcation diagram of system (4) for continuous varying $a \in(0,5)$. (b) Coexisting chaotic attractors for $a=1, b=-9$, and $\left[q_{1}, q_{2}, q_{3}\right]=[0.80,0.90,0.91]$ subject to the ICs: $\left(x_{0}, y_{0}, z_{0}\right)=(-1,1,1)$ for the red plot and $\left(x_{0}, y_{0}, z_{0}\right)=(0.1,1.2,2)$ for the blue plot.

For more clarifications about this property, the reader may refer to [26]. However, in order to show the bistability within system (4), we plot the bifurcation diagrams in Figure $15(\mathrm{a})$ when $\left[q_{1}, q_{2}, q_{3}\right]=[0.8,0.90,0.91], a=1$, and $b=-9$. It is worth noting that two sets of ICs are considered to perform the simulation of this figure. The first one is $\left(x_{0}, y_{0}, z_{0}\right)=(-1,1,1)$ which has been taken into account for the red plot, while the second one is $\left(x_{0}, y_{0}, z_{0}\right)=(0.1,1.2,2)$ which has been taken into account for the blue plot. These two plots show certainly that system (4) will exhibit bistability phenomenon if the incommensurate fractional-order value is increased. For $a=1, b=-9$, and $\left[q_{1}, q_{2}, q_{3}\right]=[0.80,0.90,0.91]$, both coexisting attractors of this system are drawn in Figure 15(b) according to the two ICs, $\left(x_{0}, y_{0}, z_{0}\right)=(-1,1,1)$ for the red plot and $\left(x_{0}, y_{0}, z_{0}\right)=$ $(0.1,1.2,2)$ for the blue plot. Furthermore, the basin of attraction of system (4) can be shown in Figure 16 which corresponds to Figures 15(a) and 15(b), where the ICs shown in yellow and blue regions lead to the limit cycles and to the chaotic attractors, respectively.

\section{Variable-Boostable Attractors of the Incommensurate Fractional-Order Model}

With the aim of accomplishing the complete range of the signal's linear transformations, the offset boosting might be joined with amplitude control. It was reported in [27] that insertion of a new developed boosting controller may destroy the symmetry of the variable-boostable model. From this perspective, we will add to the system states $x, y$, and $z$ three additional controlled scalers $m, n$, and $k$, respectively. In view of this addition, system (4) will be turned into the following form:

$$
\left\{\begin{array}{l}
D^{q_{1}} x(t)=-(x(t)+m)+2 \sin (x(t)+m)+\sin (y(t)+n)+b \sin (z(t)+k), \\
D^{q_{2}} y(t)=-(y(t)+n)+b \sin (x(t)+m)+2 \sin (y(t)+n)+a \sin (z(t)+k) \\
D^{q_{3}} z(t)=-(z(t)+k)+a \sin (x(t)+m)+b \sin (y(t)+n)+2 \sin (z(t)+k) .
\end{array}\right.
$$

In the following sections, we intend to address system (6) in light of three different cases for the system parameters $a=1$ and $b=-9$. Besides, we will select the incommensurate fractional-order values as $\left[q_{1}, q_{2}, q_{3}\right]=[0.80,0.90,0.91]$. As a remark about the choice of initial conditions, it should be noted that when the system has unbounded solutions, the variable boosting should be accompanied with a modification of the initial conditions, while the initial conditions can be ignored in the systems with global attraction.

4.1. A Line of Variable Attractors. A variable chaotic attractor can be scattered along the $1 \mathrm{D}$ line especially when we are carrying out a certain control so that the offset boosting parameters take the following cases: (i) When the parameter $m$ is frequently varied and the other two parameters are kept as $n=k=0$, we will gain several variable chaotic attractors scattered on the $x$-axis as exhibited in Figure 17(a)

(ii) When the parameter $n$ is frequently varied and the other two parameters are kept as $m=k=0$, we will gain several variable chaotic attractors scattered on the $y$-axis as exhibited in Figure 17(b)

(iii) When the parameter $k$ is frequently varied and the other two parameters are kept as $m=n=0$, we will gain several variable chaotic attractors scattered on the $z$-axis as exhibited in Figure 17 (c)

4.2. A Lattice of Variable Attractors. Herein, two controlled parameters will be simultaneously adjusted and the other 


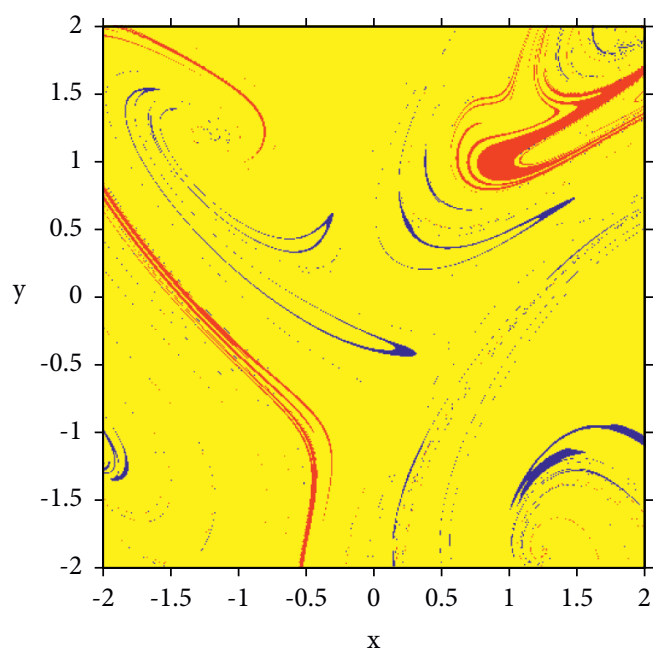

FIGURE 16: Basin of attraction of system (4) when $\left[q_{1}, q_{2}, q_{3}\right]=[0.80,0.90,0.91]$ according to the system parameters $a=1$ and $b=-9$ and to the initial condition of the third state variable $z=0$.

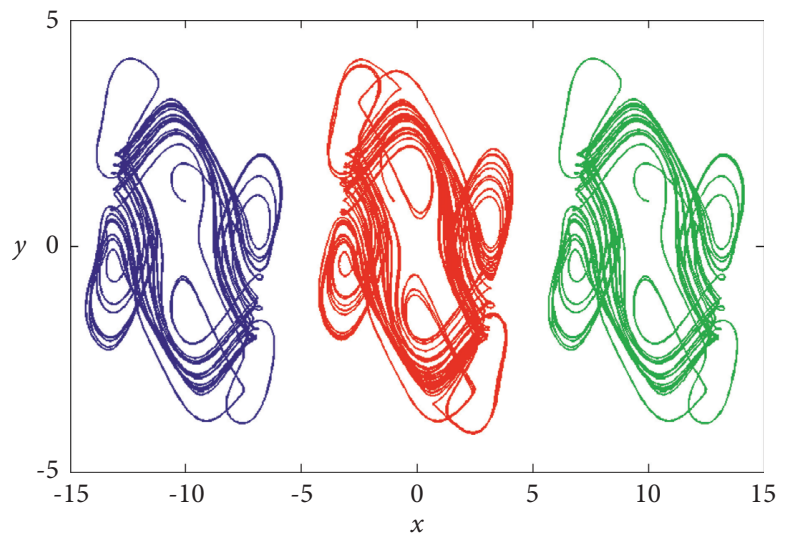

(a)

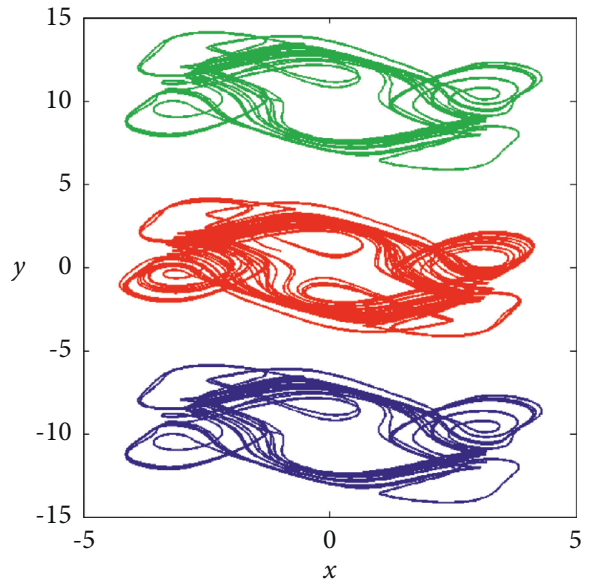

(b)

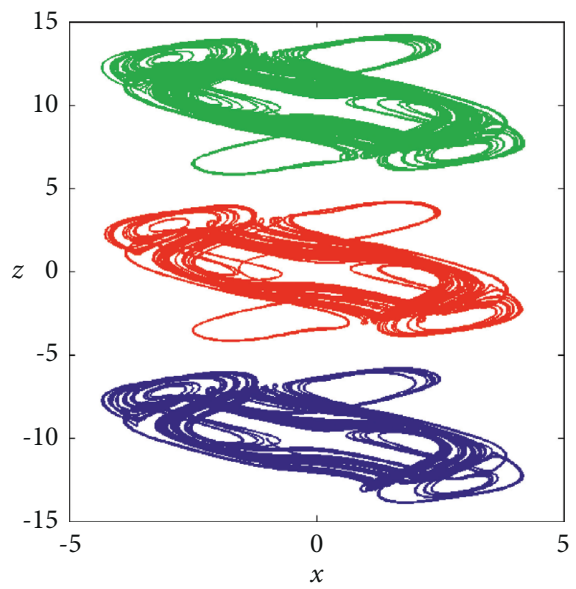

(c)

FIGURE 17: Scattering of the variable chaotic attractor on a $1 \mathrm{D}$ line for $a=1, b=-9$, and $\left[q_{1}, q_{2}, q_{3}\right]=[0.80,0.90,0.91]$. (a) $x$-line when $m= \pm 10$ and $m=0$, (b) $y$-line when $n= \pm 10$ and $n=0$, and (c) $z$-line when $k= \pm 10$ and $k=0$. 


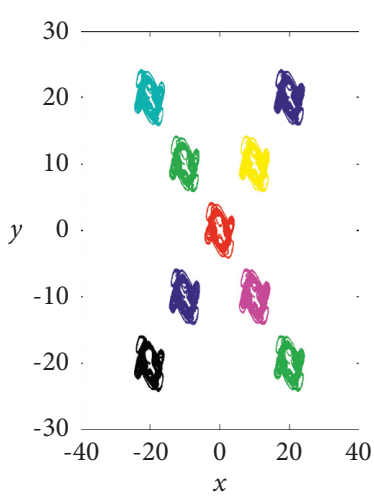

(a)

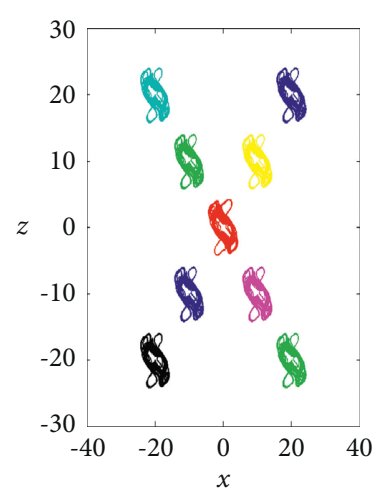

(b)

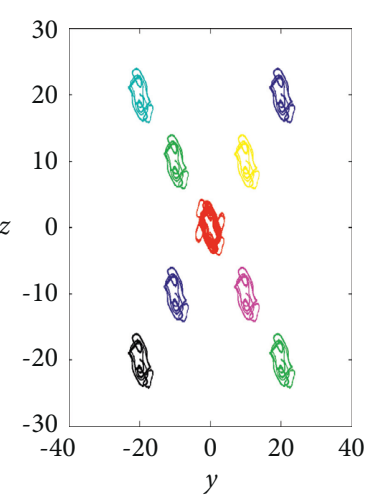

(c)

Figure 18: A 2D lattice of a variable attractor for incommensurate fractional order $\left[q_{1}, q_{2}, q_{3}\right]=[0.80,0.90,0.91]$ with $a=1$ and $b=-9$. (a) $x y$ lattice for $(m, n)=(0,0),(10,10),(10,-10),(-10,10),(-10,-10),(20,20),(20,-20),(-20,20),(-20,-20)$; (b)xz lattice for $(m, k)=(1,1),(10,10),(10,-10),(-10,10),(-10,-10),(20,20),(20,-20),(-20,20),(-20,-20)$; and $(c) y z$ lattice for $(n, k)=(1,1)$, $(10,10),(10,-10),(-10,10),(-10,-10),(20,20),(20,-20),(-20,20),(-20,-20)$.

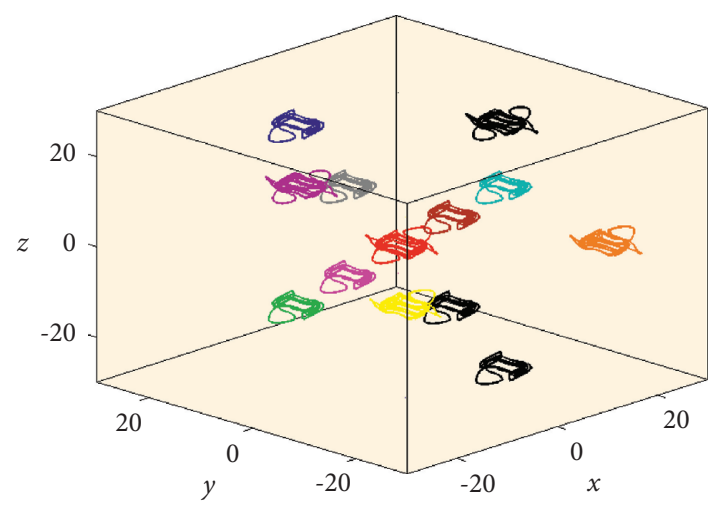

FIGURE 19: A 3D grid of a variable periodic and chaotic attractors when $\left[q_{1}, q_{2}, q_{3}\right]=[0.80,0.90,0.91]$ with $a=1$ and $b=-9$ for $(m, n, k)=(0,0,0), \quad(0,10,10), \quad(0,10,-10), \quad(0,-10,10)$, $(0,-10,-10),(0,20,20),(0,20,-20),(0,-20,20),(20,20,-20)$, $(-20,0,-20),(20,0,-20),(20,20,0),(-20,20,0)$.

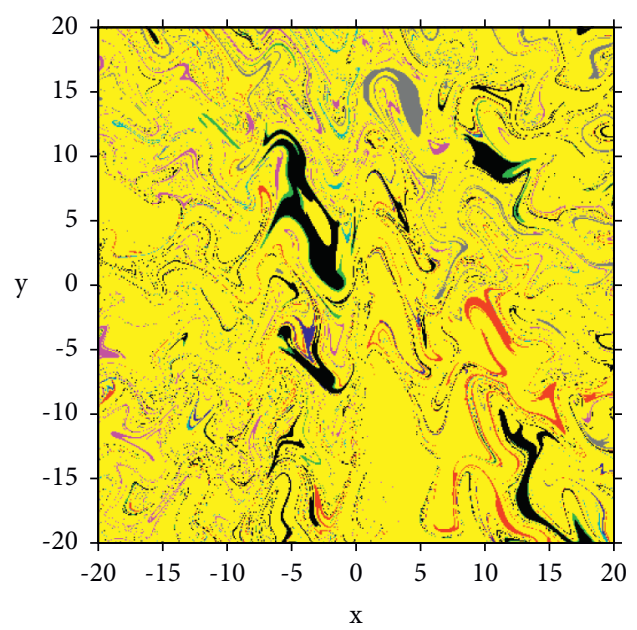

Figure 20: Basin of attraction of system (4) when $\left[q_{1}, q_{2}, q_{3}\right]=$ $[0.80,0.90,0.91]$ according to the system parameters $a=1$ and $b=$ -9 and to the initial condition of the third state variable $z=0$. The colors in this figure associate with the colors of the attractors given in Figure 19. parameter will be kept at zero. This would yield a $2 \mathrm{D}$ lattice of variable chaotic and periodic attractors. In particular, one might consider the following three cases:

(i) When the two parameters $m$ and $n$ are frequently varied and the third parameter is kept as $k=0$, we will gain several variable chaotic attractors scattered on the $x y$ lattice as exhibited in Figure 18(a)

(ii) When the two parameters $m$ and $k$ are frequently varied and the third parameter is kept as $n=0$, we will gain several variable chaotic attractors scattered on the $x z$ lattice as exhibited in Figure 18(b)

(iii) When the two parameters $n$ and $k$ are frequently varied and the third parameter is kept as $m=0$, we will gain several variable chaotic attractors scattered on the $y z$ lattice as exhibited in Figure 18(c)

4.3. A 3D Grid of Variable Attractors. In this section, certain values of the three controlled parameters $m, n$, and $k$ will be simultaneously adapted. This would yield, after taking the fractional-order values as, e.g., $\left[q_{1}, q_{2}, q_{3}\right]=[0.80,0.90,0.91]$, a $3 \mathrm{D}$ grid of variable chaotic and periodic attractors as illustrated in Figure 19. Furthermore, the basin of attraction of system (4) is plotted and shown in Figure 20 which corresponds to Figure 19, where the ICs shown in yellow and blue regions lead to the limit cycles and to the two chaotic attractors, respectively.

\section{Conclusion}

This work has formulated a novel version of Hopfield neural network models with incommensurate fractional orders using the Caputo differential operator. Through continuous variation of the values of the system parameters as well as the fractional-order derivative values, the stability of the proposed model has been analyzed numerically, and many rich complex dynamics, including symmetry, bistability, and coexisting chaotic attractors, 
have been generated. It turned out that through adapting certain additional controlled constants the proposed model possesses the offset boosting of three variables. In addition, it has been shown that the resultant periodic and chaotic attractors generated from such models can be distributed in several forms, including $1 \mathrm{D}$ line, 2D lattice, and $3 \mathrm{D}$ grid and even in an arbitrary location of the phase space.

\section{Data Availability}

The data that support the findings of this study are available upon request to the corresponding author.

\section{Conflicts of Interest}

The authors declare that they have no conflicts of interest.

\section{Acknowledgments}

The research was supported by the Taif University Researchers Supporting Project Number TURSP-2020/229, Taif University, Taif, Saudi Arabia.

\section{References}

[1] J. Fleck, "Development and establishment in artificial intelligence," The Question of Artificial Intelligence, vol. 16, pp. 106-164, 2018.

[2] S. Hayman, "The McCulloch-Pitts model,"vol. 6, pp. 4438-4439, in Proceedings of the 1999 International Joint Conference on Neural Networks (IJCNN'99), vol. 6, pp. 4438-4439, IEEE, Washington, DC, USA, 1999.

[3] S. Zhang, Y. Yu, and H. Wang, "Mittag-Leffler stability of fractional-order Hopfield neural networks," Nonlinear Analysis: Hybrid Systems, vol. 16, pp. 104-121, 2014.

[4] J. J. Hopfield, "Neural networks and physical systems with emergent collective computational abilities," Proceedings of the National Academy of Sciences, vol. 79, no. 8, pp. 25542558, 1982.

[5] I. M. Batiha, R. B. Albadarneh, S. Momani, and I. H. Jebril, "Dynamics analysis of fractional-order Hopfield neural networks," International Journal of Biomathematics, vol. 13, no. 8, Article ID 2050083, 2020.

[6] H.-P. Hu, J.-K. Wang, and F.-L. Xie, "Dynamics analysis of a new fractional-order Hopfield neural network with delay and its generalized projective synchronization," Entropy, vol. 21, no. 1, p. 1, 2019.

[7] H. Wang, Y. Yu, G. Wen, S. Zhang, and J. Yu, "Global stability analysis of fractional-order Hopfield neural networks with time delay," Neurocomputing, vol. 154, pp. 15-23, 2015.

[8] A. Boroomand and M. B. Menhaj, "Fractional-order Hopfield neural networks," Lecture Notes in Computer Science, Springer, vol. 5506, pp. 883-890, , Berlin, Germany, 2009.

[9] E. Kaslik and S. Sivasundaram, "Nonlinear dynamics and chaos in fractional-order neural networks," Neural Networks, vol. 32, pp. 245-256, 2012.

[10] B. N. Lundstrom, M. H. Higgs, W. J. Spain, and A. L. Fairhall, "Fractional differentiation by neocortical pyramidal neurons," Nature Neuroscience, vol. 11, pp. 1335-1342, 2008.

[11] P. Arena, L. Fortuna, and D. Porto, "Chaotic behavior in noninteger-order cellular neural networks," Physical Review E, vol. 61, no. 1, pp. 776-781, 2000.
[12] T. Matsuzaki and M. Nakagawa, "A chaos neuron model with fractional differential equation," Journal of the Physical Society of Japan, vol. 72, no. 10, pp. 2678-2684, 2003.

[13] I. Petras, "A note on the fractional-order cellular neural networks," in Proceedings of the 2006 IEEE International Joint Conference on Neural Network, pp. 1021-1024, Vancouver, Canada, 2006.

[14] H. Zhu, S. Zhou, and W. Zhang, "Chaos and synchronization of time delayed fractional neuron network system," in Proceedings of the 2008 9th International Conference for Young Computer Scientists, pp. 2937-2941, Changsha, China, 2008.

[15] S. Zhou, H. Li, and Z. Zhu, "Chaos control and synchronization in a fractional neuron network system," Chaos, Solitons \& Fractals, vol. 36, no. 4, pp. 973-984, 2008.

[16] S. Zhou, P. Hu, and H. Li, "Chaotic synchronization of a fractional neuron network system with time-varying delays," in Proceedings of the 2009 International Conference on Communications, Circuits and Systems, pp. 863-867, Milpitas, CA, USA, 2009.

[17] R.-C. Wu, X.-D. Hei, and L.-P. Chen, "Finite-time stability of fractional-order neural networks with delay," Communications in Theoretical Physics, vol. 60, no. 2, pp. 189-193, 2013.

[18] J. Yu, C. Hu, and H. Jiang, " $\alpha$-stability and $\alpha$-synchronization for fractional-order neural networks," Neural Networks, vol. 5, pp. 82-87, 2012.

[19] L. Chen, Y. Chai, and R. Wu, "Linear matrix inequality criteria for robust synchronization of uncertain fractional-order chaotic systems," Chaos: An Interdisciplinary Journal of Nonlinear Science, vol. 21, no. 4, Article ID 043107, 2011.

[20] I. Podlubny, Fractional Differential Equations, Academic Press, New York, NY, USA, 1999.

[21] K. Diethelm, N. J. Ford, and A. D. Freed, "A predictor-corrector approach for the numerical solution of fractional differential equations," Nonlinear Dynamics, vol. 29, pp. 3-22, 2002.

[22] D. Baleanu, K. Diethelm, E. Scalas, and J. Trujillo, Fractional Calculus Models and Numerical Methods, World Scientific Publishing Company, Singapore, 2012.

[23] K. Diethelm, "An algorithm for the numerical solution of differential equations of fractional order," Electronic Transactions on Numerical Analysis, vol. 5, pp. 1-6, 1997.

[24] K. Diethelm and N. J. Ford, "Analysis of fractional differential equations," Journal of Mathematical Analysis and Applications, vol. 265, no. 2, pp. 229-248, 2002.

[25] C. Li, J. C. Sprott, and H. Xing, "Constructing chaotic systems with conditional symmetry," Nonlinear Dynamics, vol. 87, no. 2, pp. 1351-1358, 2017.

[26] N. Debbouche, S. Momani, A. Ouannas et al., "Generating multidirectional variable hidden attractors via newly commensurate and incommensurate non-equilibrium fractionalorder chaotic systems," Entropy, vol. 23, no. 3, p. 261, 2021.

[27] C. Li and J. C. Sprott, "Variable-boostable chaotic flows," Optik, vol. 127, no. 22, pp. 10389-10398, 2016. 